\title{
Mirmecofauna em áreas de preservação permanente e plantios florestais no noroeste do Rio Grande do Sul
}

\author{
Ant fauna in permanent preservation areas and in forest plantations in the \\ northwestern region of Rio Grande do Sul state
}

\author{
Angela Maria Rizzotto ${ }^{\mathrm{II}}$, Aline Holtermann Roani ${ }^{\mathrm{I}}$, Carin Guarda ${ }^{\mathrm{II}}$, Ricardo \\ Giovenardi ${ }^{\mathrm{III}}$, Junir Antonio Lutinski ${ }^{\mathrm{IV}}$
}

\begin{abstract}
Resumo
As formigas compreendem um táxon muito diverso de insetos, apresentam elevada riqueza e são ecologicamente importantes nos diferentes ecossistemas terrestres. A riqueza e diversidade de formigas tendem a aumentar de acordo com a complexidade dos ambientes no que se refere à vegetação e ao estágio de conservação da biodiversidade. Este estudo visou caracterizar as assembleias de formigas que ocorrem em duas áreas de preservação permanente, descritas como fragmentos de vegetação nativa circundados por lavouras e, dois plantios de eucalipto, cercados por lavouras e pastagens. O estudo foi realizado no município de Frederico Westphalen ( $27^{\circ} 21^{\prime} 27^{\prime \prime} ; 53^{\circ} 23^{\prime} 40^{\prime \prime}$ ), noroeste do Rio Grande do Sul. Foram utilizadas 10 armadilhas de queda (pitfall) e 20 iscas, 10 de sardinha e 10 de glicose em cada área. A riqueza foi definida como o número de espécies que ocorreram em cada uma das amostras e foi comparada por meio de análise de rarefação baseadas no número de ocorrências. A relação entre as espécies de formigas e os diferentes ambientes foi verificada através da Análise de Componentes Principais (PCA). A riqueza de formigas amostrada foi de 71 espécies, pertencentes a 19 gêneros, cinco subfamílias e, sete espécies ocorreram nos quatro ambientes amostrados. A subfamília Myrmicinae e os gêneros Pheidole e Camponotus foram os mais ricos no estudo. Observou-se diferença significativa quanto à riqueza, abundância e a composição das assembleias de formigas entre os ambientes amostrados. Os resultados apontam diferenças entre as assembleias de formigas dos fragmentos florestais e das plantações de eucalipto, e acrescentam informações acerca da biodiversidade da região do estudo.
\end{abstract}

Palavras-chave: Bioindicadores ecológicos; Conservação da biodiversidade; Entomologia florestal; Pheidole

\section{Abstract}

The ants comprise a very diverse taxon of insects, present high richness and are ecologically important in the different terrestrial ecosystems. The richness and diversity of ants tend to increase according to the complexity of the environments in terms of vegetation and the stage of biodiversity conservation. This study aimed at inventorying and characterizing ant assemblages that occur in two permanent preservation areas, characterized as fragments of native vegetation surrounded by crops, and two eucalyptus plantations, surrounded by crops and pastures. The study was carried out in the municipality of Frederico Westphalen ( $27^{\circ} 21^{\prime} 27^{\prime \prime}, 53^{\circ} 23^{\prime} 40^{\prime}$ W), located in the northwestern region of Rio Grande do Sul state. Ten pitfall traps and 20 baits, 10 sardines and 10 glucose ones were used in each area. The richness was defined as the number of species that occurred in each sample and was compared by means of rarefaction analysis based on the number of occurrences. The relationship between the ant species and the different environments was verified through a Principal Component Analysis (PCA). The richness of ants was 71 species, belonging to 19 genera and five subfamilies. Seven species occurred in four sampled environments. The subfamily Myrmicinae and the genera Pheidole and Camponotus were the richest ones in the study. A significant difference was observed regarding the richness, abundance and composition of ant assemblages among the sampled environments. A total of $76.4 \%$ of the variation in the occurrence of ants, according to the environments sampled, was explained by the first two components of the PCA. The results point out differences between ant assemblages from forest fragments and those from eucalyptus plantations. They add information about the biodiversity of the study region.

Keywords: Ecological bioindicators; Biodiversity conservation; Forest entomology; Pheidole

Biólogas, Esp., Programa de Pós-Graduação em Conservação da Biodiversidade, Universidade Regional Integrada do Alto Uruguai e das Missões, Rua Assis Brasil, 709, CEP 98400-000, Frederico Westphalen (RS), Brasil. angelamariarizzotto@gmail.com (ORCID: 0000-0001-5900-3239) aline@uri.edu.br (ORCID: 0000-0002-4683-4575)

Bióloga, MSc., Doutoranda do Programa de Pós-Graduação em Ciências da Saúde, Universidade Comunitária da Região de Chapecó. Servidão Anjo da Guarda, 295-D - Efapi, CEP 89809-900, Chapecó (SC), Brasil. carin@unochapeco.edu.br (ORCID: 0000-0003-4323-5080)

III Biólogo, Dr., Programa de Pós-Graduação em Conservação da Biodiversidade, Universidade Regional Integrada do Alto Uruguai e das Missões, Rua Assis Brasil, 709, CEP 98400-000, Frederico Westphalen (RS), Brasil. ricardogiovenardi@gmail.com (ORCID: 0000-0001-6833-2077)

IV Biólogo, Dr., Docente do Programa de Pós-Graduação em Ciências da Saúde, Universidade Comunitária da Região de Chapecó. Servidão Anjo da Guarda, 295-D - Efapi, CEP 89809-900, Chapecó (SC), Brasil. junir@unochapeco.edu.br (ORCID: 000-0003-0149-5415) 


\section{Introdução}

Os ecossistemas são importantes para a manutenção da biodiversidade, sobrevivência e conservação das espécies. As atividades antrópicas como a transformação de ambientes naturais para fins agrícolas, pecuária, urbanização, instalações industriais e a produção de energia geram impactos ambientais que levam às perdas e ameaças à conservação da biodiversidade (MADEIRA et al., 2009).

A transformação de ambientes naturais em áreas agrícolas levou a uma crescente preocupação com os impactos sobre as comunidades de organismos em geral. Dentre as causas desses impactos, destacam-se a fragmentação do habitat, o uso de agrotóxicos e as monoculturas (EDWARDS et al., 2014). Os processos de regeneração da vegetação podem afetar as comunidades de formigas, cuja intensidade da alteração ou degradação ambiental pode responder de diferentes formas, beneficiando algumas espécies ou prejudicando outras (HÖLLDOBLER; WILSON, 1990).

As formigas (Hymenoptera: Formicidae) compreendem um táxon diverso de insetos (HOLDEFER; LUTINSKI; GARCIA, 2017; LUTINSKI et al., 2017a), altamente funcional e especializado (HÖLLDOBLER; WILSON, 1990) caracterizado por sua abundância em ambientes terrestres, riqueza e ampla distribuição (WIELGOSS et al., 2014). Também são um componente importante dos ecossistemas, constituindo uma parte substancial da biomassa animal (NAYANA et al., 2016).

Alguns insetos são considerados indicadores ecológicos em virtude das funções que desempenham na natureza, da estreita relação com a heterogeneidade dos ecossistemas e processos ecológicos a sensibilidade às mudanças ambientais. As estruturas das assembleias de formigas vêm sendo estudadas e recebendo particular interesse, pela sua capacidade de serem bioindicadores ambientais devido a sua ampla distribuição, resposta rápida às mudanças ambientais e relevância biológica em diferentes níveis tróficos (CREPALDI et al., 2014; BHARTI; BHARTI; PFEIFFER, 2016; LUTINSKI et al., 2017a).

Formigas apresentam grande riqueza e são ecologicamente importantes nos diferentes ecossistemas terrestres (BACCARO et al., 2015), são sensíveis a impactos antropogênicos e fatores bióticos e abióticos que podem determinar a riqueza, abundância e distribuição de outros organismos (NAYANA et al., 2016). A riqueza e a diversidade de formigas tendem a aumentar de acordo com a complexidade dos ambientes (ROCHA et al., 2015; HOLDEFER; LUTINSKI; GARCIA, 2017). São úteis para avaliar as respostas da biodiversidade animal à restauração florestal. Nesse sentido, as Áreas de Preservação Permanente (APP) são consideradas determinantes para conservação da biodiversidade e, juntamente com os remanescentes de fragmentos florestais, garantem condições favoráveis para o estabelecimento e a manutenção da fauna e da flora, bem como para a manutenção do equilíbrio ecológico (TUNDISI; TUNDISI, 2010).

As formigas são relativamente fáceis de amostrar, possuem ampla distribuição geográfica, são abundantes, importantes no funcionamento dos ecossistemas e sua taxonomia e ecologia são relativamente conhecidas (ROCHA et al., 2015). Têm sido usadas em diversos trabalhos para avaliar as perturbações de habitas, o impacto do desmatamento e reflorestamento com plantas exóticas (COSTA-MILANEZ et al., 2014; CANTARELLI et al., 2015), a contaminação por pesticidas como inseticidas, herbicidas, acaricidas e fungicidas (ILHA et al., 2009), o impacto da urbanização (LUTINSKI; LOPES, B.; MORAIS, 2013), o monitoramento de áreas de mineração (ROCHA et al., 2015) e a produção de energia (LUTINSKI et al., 2017c).

Nesse contexto, o objetivo deste estudo foi caracterizar as assembleias de formigas que ocorrem em duas áreas de preservação permanente, descritas como fragmentos de vegetação nativa, circundadas por lavouras e, dois plantios de eucalipto, um com oito e outro com 15 anos, cercados por lavouras e pastagens e com sub-bosque formado por gramíneas e arbustos, localizados na região norte do Rio grande do Sul. 


\section{Material e método}

\section{Área de estudo}

O estudo foi realizado no Município de Frederico Westphalen (27²1’32”S; 5323’38”O), na região do Médio Alto Uruguai, localizado no norte do Estado do Rio Grande do Sul. Possui área territorial de aproximadamente $265 \mathrm{~km}^{2}$, inserida no bioma de Mata Atlântica (IBGE, 2016), com domínio Floresta Estacional Decidual. O estudo contemplou quatro ambientes, sendo duas Áreas de Preservação Permanente (APP1 e APP2) e dois de Reflorestamento de eucalipto (EUC1 e EUC2). O clima da região é considerado subtropical do tipo Cfa (ALVARES et al., 2014), apresentando temperatura média anual de $18^{\circ} \mathrm{C}$, com máximas no verão de $41^{\circ} \mathrm{C}$ e mínimas no inverno inferiores a $0^{\circ} \mathrm{C}$. A precipitação média anual é considerada elevada, sendo bem distribuída ao longo do ano, atingindo entre 1.800 a $2.100 \mathrm{~mm}$. A seguir apresenta-se a caracterização dos ambientes avaliados:

Ambiente 1 (APP1): Área de Preservação Permanente $\left(27^{\circ} 22^{\prime} 31.99\right.$ ”S 5325’36.95”O) com cerca de 35 ha de mata, formado por uma vegetação nativa, pouco perturbada embora seja circundado por lavouras de diversos cultivos, o fragmento apresenta um Lajeado perfazendo sua extensão, diferenciando do outro estrato de mata.

Ambiente 2 (APP2): Área de Preservação Permanente $\left(27^{\circ} 22^{\prime} 38.70^{\prime \prime S}\right.$ 53⒉ $24^{\prime} 45.08^{\prime \prime}$ ) com cerca de 50 ha de mata, formado por uma vegetação nativa perturbada pela antropização, geralmente frequentado por pessoas nos fins de semana, apresentando uma clareira em seu centro ocupada para plantações de trigo e soja e circundado por lavouras.

Ambiente 3 (EUC1): com extensão aproximada de 15 ha $\left(27^{\circ} 22^{\prime} 39.32^{\prime \prime S} 53^{\circ} 22\right.$ 16.81»O) constituída por uma plantação de eucalipto (Eucalyptus sp.), com aproximadamente 15 anos, altura média aproximada de 25 metros e diâmetro a altura do peito (DAP) aproximado de 35 $\mathrm{cm}$. Está localizada a uma distância de $7 \mathrm{~km}$ da área urbana. No entorno do ambiente há uma pastagem, pequenas moradias, lavouras e mata. O sub-bosque é composto essencialmente por gramíneas e arbustos esparsos, resultando em uma serapilheira em torno de $10 \mathrm{~cm}$.

Ambiente 4 (EUC2): com extensão aproximada de 1 ha $\left(27^{\circ} 23^{\prime} 19.83\right.$ ”'S 53 $20^{\prime} 39.67^{\prime \prime}$ ) constituída por uma plantação de eucalipto (Eucalyptus sp.), com aproximadamente oito anos, com aproximadamente 15 anos, altura média aproximada de 15 metros, diâmetro a altura do peito (DAP) aproximado de $20 \mathrm{~cm}$, entremeado por pastagem e ocupado por bovinos. Localizada a uma distância de $13 \mathrm{~km}$ da área urbana e no entono possuem lavouras, criação de gado e mata. O sub-bosque é composto essencialmente por gramíneas e arbustos esparsos, resultando em uma serapilheira pobre.

\section{Amostragem}

Uma amostragem foi realizada em cada um dos ambientes no ano de 2018. Formigas são afetadas pela temperatura e, noverão apresentam maior atividade fora da colônia (HÖLLDOBLER; WILSON, 1990). Por esta razão, a amostragem foi conduzida do mês de março. Foram instaladas 10 armadilhas de queda do tipo pitfall em cada ambiente, com $10 \mathrm{~cm}$ de diâmetro e $20 \mathrm{~cm}$ de profundidade, distribuídas em transectos lineares, perpendiculares à borda. A primeira, a cinco metros da borda e as demais, distantes $10 \mathrm{~m}$ uma da outra. As armadilhas continham uma solução de $200 \mathrm{ml}$ de água com uma gota de detergente, para quebrar a tensão superficial da água (ILHA et al., 2009), e permaneceram expostas por 48 horas.

No mesmo período de amostragem com pitfalls, também foram utilizadas 10 iscas de $1 \mathrm{~g}$ de sardinha e dez de $1 \mathrm{ml}$ de glicose, preparadas sob retângulos de papel poroso e distribuídas de forma alternada, distantes $10 \mathrm{~m}$ entre si (BESTELMEYER et al., 2000; LUTINSKI; LOPES; MORAIS, 2013). Foram estabelecidos transectos perpendiculares à margem externa e obedecida uma distância de cinco metros da borda externa (BESTELMEYER et al., 2000), e as armadilhas foram expostas por uma hora em cada ambiente, no período entre $9 \mathrm{~h}$ e $17 \mathrm{~h}$. 
Os espécimes amostrados foram devidamente acondicionados e identificados em frascos e transportados para o Laboratório de Morfologia da Universidade Regional Integrada do Alto Uruguai e das Missões - Campus de Frederico Westphalen, para triagem e identificação em morfoespécies. Após, foram identificadas com base nas chaves taxonômicas propostas por Fernandez (2003) e Baccaro et al. (2015). Exemplares se encontram depositados no Laboratório de Morfologia da Universidade Regional Integrada do Alto Uruguai e das Missões - Campus de Frederico Westphalen-RS, Brasil.

\section{Análise dos dados}

A riqueza foi definida como o número de espécies que ocorreram em cada uma das amostras e a abundância com base na frequência de ocorrência (ou seja, o número de registros de uma dada espécie em cada armadilha ou isca e em ambiente) e não no número de indivíduos, o que é mais apropriado para os estudos com assembleias de formigas, minimizando assim o efeito dos hábitos de forrageio e o tamanho das colônias (ROMERO; JAFFE, 1989). A frequência relativa de ocorrência de cada espécie em cada ambiente amostrado foi calculada pela seguinte equação: $\mathrm{f}(\%)=\mathrm{Fi} \times 100 / \mathrm{Ft}$, em que Fi é o número de ocorrências de uma determinada espécie em cada armadilha ou isca, em um determinado ambiente e Ft é o número total de ocorrências para este determinado ambiente (LUTINSKI et al., 2017a).

A riqueza de formigas de cada ambiente foi comparada por meio de uma análise de rarefação baseada no número de ocorrências. Essa análise foi realizada com o auxílio do programa EcoSim 7 (GOTELLI; ENTSMINGER, 2001) e permite comparar conjuntos de amostras.

Para verificar a riqueza estimada, foi realizada uma comparação entre as espécies observadas (Sobs) e a riqueza estimada pelo estimador não paramétrico Chao 1. Esta comparação permite inferir quanto uma amostragem se aproxima de todas as espécies existentes em um determinado ambiente. As estimativas de riqueza para cada ambiente foram obtidas utilizando o software PAST (HAMMER; HARPER; RYAN, 2001).

A avaliação da diversidade (riqueza e abundância) foi realizada utilizando o índice de diversidade de Shannon. Este índice permite estimar a diversidade de espécies locais. Foi avaliado o índice de Equitabilidade de Pielou (J'), o qual permite representar a uniformidade de distribuição dos indivíduos entre todas as espécies existentes. Ambas as análises foram obtidas utilizando o software PAST (HAMMER; HARPER; RYAN, 2001).

Foi feita uma ordenação NMDS (Non-metric Multidimensional Scaling) para testar se a abundância e a composição das assembleias de formigas diferem nos ambientes amostrados e nos métodos utilizados. A matriz dos dados foi previamente transformada em $\log (\mathrm{x}+1)$, foi utilizado Bray-Curtis como índice de associação e a análise foi realizada com o programa estatístico Primer 6.1.9 (CLARKE; GORLEY, 2005).

A relação entre as espécies de formigas e os ambientes foi verificada através da Análise de Componentes Principais (PCA). Quarenta e quatro espécies de formigas foram excluídas da análise devido ao número de ocorrências nas amostras $(\leq 2)$. Os dados foram previamente transformados em $\log (x+1)$ e analisados usando o software PAST (HAMMER; HARPER; RYAN, 2001).

O estudo foi autorizado pelo Instituto Chico Mendes de Conservação da Biodiversidade (ICMBio) mediante autorização de “autorização para atividades científicas”: n 61104-1.

\section{Resultados e discussão}

Foram amostrados 6.771 espécimes de formigas, distribuídos em 71 espécies, pertencentes a 19 gêneros e a cinco subfamílias. A subfamília Myrmicinae foi a mais rica $(S=40)$, seguida de Formicinae $(S=23)$ e Dolichoderinae $(S=4)$. O gênero Pheidole foi o mais rico, com 23 espécies, seguido por Camponotus ( $\mathrm{S}=15)$, Brachymyrmex ( $\mathrm{S}=5)$, Crematogaster, Linepithema e Solenopsis $(\mathrm{S}=4)$.

Ci. Fl., Santa Maria, v. 29, n. 3, p. 1227-1240, jul./set. 2019 
Sete espécies foram registradas nos quatro ambientes amostrados: Acromyrmex subterraneus (Forel, 1893), Pachycondyla striata (F. Smith, 1858), Pheidole dyctiota (Kempf, 1972), Pheidole megacephala (Fabricius, 1793), Pheidole risii (Forel, 1892), Pheidole sp. 1 e Pheidole sp. 8 e 46 (65 \%) espécies ocorreram de forma exclusiva (Tabela 1).

A assembleia do ambiente APP1 teve como espécies mais frequentes Pheidole aberrans (Mayr, 1868), Pheidole risii, e Pheidole sp. 12; no ambiente APP2 as espécies Pheidole risii, Pheidole sp. 8 e Pheidole striata; no EUC1, as espécies Camponotus rufipes (Fabricius, 1775), Pheidole dyctiota, Pheidole pubiventris (Mayr, 1887), Pheidole risii, e Pheidole sp. 8; no EUC2 Pheidole risii, Pheidole sp. 1 e Pheidole sp. 8 (Tabela 1).

Tabela 1 - Frequência das espécies de formigas amostradas nos quatro ambientes no município de Frederico Westphalen, Rio Grande do Sul, Brasil (2018).

Table 1 - Ant frequency sampled in the four environments in Frederico Westphalen municipality, Rio Grande do Sul state, Brazil (2018).

\begin{tabular}{|c|c|c|c|c|c|c|c|c|}
\hline \multirow{2}{*}{ Táxon } & \multicolumn{2}{|c|}{ APP1 } & \multicolumn{2}{|c|}{ APP2 } & \multicolumn{2}{|c|}{ EUC1 } & \multicolumn{2}{|c|}{ EUC2 } \\
\hline & $\mathbf{N}$ & $\mathbf{f}(\%)$ & $\mathbf{n}$ & $\mathbf{f}(\%)$ & $\mathbf{n}$ & $f(\%)$ & $\mathbf{N}$ & $\mathbf{f}(\%)$ \\
\hline \multicolumn{9}{|l|}{ Subfamília Dolichoderinae } \\
\hline Linepithema angulatum (Emery, 1894) & 6 & 5,41 & 2 & 2,30 & & & 7 & 6,36 \\
\hline Linepithema humile (Mayr, 1868) & & & 1 & 1,15 & 2 & 2,33 & & \\
\hline Linepithema sp. 1 & & & 1 & 1,15 & & & & \\
\hline Linepithema sp. 2 & 3 & 2,70 & & & & & & \\
\hline \multicolumn{9}{|l|}{ Subfamília Formicinae } \\
\hline Brachymyrmex aphidicola (Forel, 1909) & 5 & 4,50 & 1 & 1,15 & & & 5 & 4,55 \\
\hline Brachymyrmex coactus (Mayr, 1887) & & & & & 1 & 1,16 & 2 & 1,82 \\
\hline Brachymyrmex sp. 1 & & & & & & & 1 & 0,91 \\
\hline Brachymyrmex sp. 2 & & & & & & & 2 & 1,82 \\
\hline Brachymyrmex sp. 3 & 1 & 0,90 & & & & & & \\
\hline Camponotus blandus (F. Smith, 1858) & & & & & 2 & 2,33 & & \\
\hline Camponotus crassus (Mayr, 1862) & & & & & 3 & 3,49 & & \\
\hline Camponotus diversipalpus (Santschi, 1922) & & & & & 2 & 2,33 & & \\
\hline Camponotus lespesii (Forel, 1886) & & & & & 1 & 1,16 & & \\
\hline Camponotus mus (Roger, 1863) & & & & & & & 1 & 0,91 \\
\hline Camponotus rufipes (Fabricius, 1775) & 4 & 3,60 & 2 & 2,30 & 6 & 6,98 & & \\
\hline Camponotus sericeiventris (G.-Mén., 1838) & & & 1 & 1,15 & & & & \\
\hline Camponotus sp. 1 & & & & & 2 & 2,33 & & \\
\hline Camponotus sp. 2 & & & & & 5 & 5,81 & & \\
\hline Camponotus sp. 3 & & & 1 & 1,15 & 1 & 1,16 & & \\
\hline Camponotus sp. 4 & & & & & 1 & 1,16 & & \\
\hline
\end{tabular}


Tabela 1 - Continuação...

Table 1 - Continuation...

\begin{tabular}{|c|c|c|c|c|c|c|c|c|}
\hline \multirow{2}{*}{ Táxon } & \multicolumn{2}{|c|}{ APP1 } & \multicolumn{2}{|c|}{ APP2 } & \multicolumn{2}{|c|}{ EUC1 } & \multicolumn{2}{|c|}{ EUC2 } \\
\hline & $\mathbf{N}$ & $\mathbf{f}(\%)$ & $\mathbf{n}$ & $\mathbf{f}(\%)$ & $\mathbf{n}$ & $f(\%)$ & $\mathbf{N}$ & $\mathrm{f}(\%)$ \\
\hline Camponotus sp. 5 & & & & & 1 & 1,16 & 1 & 0,91 \\
\hline Camponotus sp. 6 & & & & & & & 1 & 0,91 \\
\hline Camponotus sp. 7 & 1 & 0,90 & & & & & & \\
\hline Camponotus sp. 8 & 1 & 0,90 & & & & & & \\
\hline Nylanderia fulva (Mayr, 1862) & 1 & 0,90 & & & 3 & 3,49 & & \\
\hline Nylanderia sp. & & & & & 1 & 1,16 & & \\
\hline Paratrechina longicornis (Latreille, 1802) & 1 & 0,90 & 4 & 4,60 & & & & \\
\hline \multicolumn{9}{|l|}{ Subfamília Myrmicinae } \\
\hline Acromyrmex subterraneus (Forel, 1893) & 5 & 4,50 & 4 & 4,60 & 2 & 2,33 & 3 & 2,73 \\
\hline Apterostigma mayri (Forel, 1893) & & & 1 & 1,15 & & & & \\
\hline Crematogaster corticicola (Mayr, 1887) & 2 & 1,80 & & & & & 1 & 0,91 \\
\hline Crematogaster magnifica (Santschi, 1925) & & & & & 1 & 1,16 & & \\
\hline Crematogaster sp. 1 & 1 & 0,90 & & & & & & \\
\hline Crematogaster sp. 2 & 2 & 1,80 & & & & & & \\
\hline Cyphomyrmex rimosus (Spinola, 1853) & 3 & 2,70 & & & & & & \\
\hline Cyphomyrmex strigatus (Mayr, 1887) & & & 1 & 1,15 & & & & \\
\hline Monomorium floricola (Jerdon, 1851) & 1 & 0,90 & 1 & 1,15 & & & & \\
\hline Mycocepurus goeldii (Forel, 1893) & 3 & 2,70 & 1 & 1,15 & & & & \\
\hline Pheidole aberrans (Mayr, 1868) & 13 & 11,71 & & & 3 & 3,49 & 2 & 1,82 \\
\hline Pheidole dyctiota (Kempf, 1972) & 2 & 1,80 & 5 & 5,75 & 6 & 6,98 & 6 & 5,45 \\
\hline Pheidole laevifrons (Mayr, 1887) & & & & & 1 & 1,16 & & \\
\hline Pheidole megacephala (Fabricius, 1793) & 3 & 2,70 & 4 & 4,60 & 1 & 1,16 & 7 & 6,36 \\
\hline Pheidole pubiventris (Mayr, 1887) & 1 & 0,90 & & & 6 & 6,98 & 9 & 8,18 \\
\hline Pheidole risii Forel, 1892 & 21 & 18,92 & 16 & 18,39 & 6 & 6,98 & 17 & 15,45 \\
\hline Pheidole sp. 1 & 3 & 2,70 & 3 & 3,45 & 1 & 1,16 & 14 & 12,73 \\
\hline Pheidole sp. 2 & & & & & 1 & 1,16 & & \\
\hline Pheidole sp. 3 & & & & & 2 & 2,33 & & \\
\hline Pheidole sp. 4 & & & & & 1 & 1,16 & & \\
\hline Pheidole sp. 5 & & & & & 1 & 1,16 & & \\
\hline Pheidole sp. 6 & & & & & 2 & 2,33 & & \\
\hline Pheidole sp. 7 & & & & & 1 & 1,16 & & \\
\hline Pheidole sp. 8 & 5 & 4,50 & 19 & 21,84 & 7 & 8,14 & 12 & 10,91 \\
\hline Pheidole sp. 9 & & & & & 1 & 1,16 & & \\
\hline Pheidole sp. 10 & & & & & 1 & 1,16 & & \\
\hline
\end{tabular}


Tabela 1 - Conclusão...

Table 1 - Conclusion...

\begin{tabular}{|c|c|c|c|c|c|c|c|c|}
\hline \multirow{2}{*}{ Táxon } & \multicolumn{2}{|c|}{ APP1 } & \multicolumn{2}{|c|}{ APP2 } & \multicolumn{2}{|c|}{ EUC1 } & \multicolumn{2}{|c|}{ EUC2 } \\
\hline & $\mathbf{N}$ & $f(\%)$ & n & $f(\%)$ & $\mathbf{n}$ & $f(\%)$ & $\mathbf{N}$ & $f(\%)$ \\
\hline Pheidole sp. 11 & & & & & 2 & 2,33 & & \\
\hline Pheidole sp. 12 & 7 & 6,31 & & & 1 & 1,16 & 4 & 3,64 \\
\hline Pheidole sp. 13 & & & 2 & 2,30 & & & & \\
\hline Pheidole sp. 14 & & & & & & & 1 & 0,91 \\
\hline Pheidole sp. 15 & & & & & & & 1 & 0,91 \\
\hline Pheidole sp. 16 & 1 & 0,90 & & & & & 7 & 6,36 \\
\hline Pheidole sp. 17 & 3 & 2,70 & & & & & & \\
\hline Solenopsis saevissima (F. Smith, 1855) & 1 & 0,90 & & & & & & \\
\hline Solenopsis stricta Emery, 1896 & & & & & 2 & 2,33 & 1 & 0,91 \\
\hline Solenopsis sp. 1 & & & & & 1 & 1,16 & & \\
\hline Solenopsis sp. 2 & & & 1 & 1,15 & & & & \\
\hline Solenopsis sp. 3 & 2 & 1,80 & & & & & & \\
\hline Wasmannia auropunctata (Roger, 1863) & 1 & 0,90 & & & 1 & 1,16 & & \\
\hline Wasmannia sp. & & & & & & & 2 & 1,82 \\
\hline \multicolumn{9}{|l|}{ Subfamília Ponerinae } \\
\hline Hypoponera sp. 1 & & & 1 & 1,15 & & & & \\
\hline Odontomachus chelifer (Latreille, 1802) & & & & & 1 & 1,16 & & \\
\hline Pachycondyla striata (F. Smith, 1858) & 4 & 3,60 & 15 & 17,24 & 3 & 3,49 & 3 & 2,73 \\
\hline \multicolumn{9}{|l|}{ Subfamília Pseudomyrmecinae } \\
\hline Pseudomyrmex gracilis (Fabricius, 1804) & 4 & 3,60 & & & & & & \\
\hline
\end{tabular}

Em que: APP1-2: Áreas de preservação permanente, 1 e 2; EUC1-2: Plantações de eucalipto, 1 e 2. n: frequência de ocorrência; f: frequência relativa.

A fauna de formigas (gêneros e espécies) encontrada neste estudo é similar à amostrada em outras pesquisas já realizadas na região sul do Brasil. As 71 epécies amostradas representam $65,7 \%$ da fauna de formigas registradas por Cantarelli et al. (2015) para ambientes na região Noroeste do Rio Grande do Sul, e 34,3\% das espécies descritas para a região oeste do estado de Santa Catarina (ULYSSÉA et al., 2011; LUTINSKI et al., 2018). Apesar dos estudos já existentes, este trabalho amplia o conhecimento da mirmecofauna para uma região ainda pouco amostrada do estado do Rio Grande Sul. Ainda, aponta para a distribuição e para a diversidade de formigas que ocorrem em ambientes em processo de regeneração ecológica.

As assembleias de formigas geralmente incluem subfamílias e gêneros reconhecidos por desempenharem papéis importantes no vários níveis tróficos do ambiente amostrado (HÖLLDOBLER; WILSON, 1990) e incluem táxons que são frequentemente encontrados em estudos regionais (ILHA et al., 2009; ULYSSÉA et al., 2011; LUTINSKI; LOPES; MORAIS, 2013; CANTARELLI et al., 2015; LUTINSKI et al., 2017a). A subfamília Myrmicinae foi a mais rica no presente estudo. É considerada dominante nos biomas brasileiros, como a Mata Atlântica e o Cerrado, em ambos os casos, em número de gêneros e espécies. Possuem algumas características notáveis, incluindo a diversidade de hábitos alimentares e de nidificação o que explicam seu 
sucesso (HÖLLDOBLER; WILSON, 1990).

Os gêneros mais ricos nas amostras foram Pheidole e Camponotus, representando 32,4 e 21,1\% da riqueza, respectivamente. Este resultado está de acordo com os mais recentes e representativos levantamentos de fauna de formigas na região Noroeste do Estado do Rio Grande do Sul, realizado por Cantarelli et al. (2015) e na região oeste de Santa Catarina, realizados por Lutinski, Lopes e Morais (2013) e Lutinski et al. (2018). Ambos os gêneros se caracterizam por serem dominantes, generalistas, oportunistas e onívoros (SILVESTRE; BRANDÃO; SILVA, 2003; BACCARO et al., 2015).

$\mathrm{O}$ gênero Camponotus, é considerado o maior gênero de formigas. Dominantes onde ocorrem, as espécies desse gênero desempenham importante papel ecológico. A maioria é dimórfica ou polimórfica e arborícola (BACCARO et al., 2015). O gênero Pheidole é considerado hiperdiverso, cosmopolita e pode ser encontrado nos mais diversos ambientes. Algumas espécies auxiliam na dispersão de sementes. Por sua diversidade e abundância possuem grande importância ecológica como controladoras das populações de outros artrópodes. Formigas são consideradas bioindicadores e Camponotus e Pheidole são frequentemente encontradas em ambientes degradados (SILVESTRE; BRANDÃO; SILVA, 2003). A riqueza das formigas desses gêneros pode explicar a riqueza amostrada como um todo nos quatro ambientes, considerando-se que os ambientes EUC1 e EUC2 apresentaram diversidade comparável ou mesmo maior que as APP.

A riqueza e a ocorrência do gênero Acromyrmex, Pachycondyla e Pheidole em todos os ambientes amostrados demonstram a importância destas formigas nos ambientes. A ocorrência de formigas cortadeiras como Acromyrmex subterraneus, pode ser explicada pela cobertura vegetal, de onde obtêm folhas e outras partes vegetais para cultivar o fungo que serve como alimento (SILVESTRE; BRANDÃO; SILVA, 2003). Formigas deste gênero constroem seus ninhos subterrâneos e são consideradas pragas agrícolas, causando danos às pastagens, cultivos de cana-de-açúcar, eucalipto e jardins (BACCARO et al., 2015). Formigas do gênero Pachycondyla são encontradas nos mais diversos ambientes, especialmente em regiões de florestas. Forrageiam e constroem seus ninhos no solo, são predadoras generalistas e ocasionalmente se alimentam do arilo de sementes caídas no solo das florestas. Podem forragear solitárias ou fazer recrutamento (BACCARO et al., 2015). A ocorrência de formigas Pachycondyla pressupõe a ocorrência de uma fauna de invertebrados de solo e de serapilheira, o que indica a existência dessas condições em todos os ambientes amostrados.

A ocorrência de espécies dos gêneros Apterostigma, Cyphomyrmex, Hypoponera e Odontomachus também ressaltam a importância do processo de regeneração e a formação de serapilheira. Esta serve de abrigo para essas formigas (SILVESTRE; BRANDÃO; SILVA, 2003). Hypoponera e Odontomachus são predadores especializados que caçam suas presas (invertebrados) no solo, constroem seus ninhos em troncos caídos ou sob pedras. Eles são identificados por possuírem baixa agilidade e formarem pequenas colônias. Têm afinidade com ambientes sombreados e úmidos. Apterostigma e Cyphomyrmex utilizam matéria orgânica em decomposição para o cultivo de fungos que usam como alimento (SILVESTRE; BRANDÃO; SILVA, 2003), explicando as ocorrências dessas espécies somente na APP1 e APP2.

Formigas do gênero Linepithema são encontradas em ambientes antropizados e ocorrem frequentemente em diversos ambientes do sul do Brasil (ULYSSÉA et al., 2011). Algumas das espécies que pertencem a este gênero são consideradas pragas em ambientes urbanos, destacandose a espécie Linepithema humile (FERNANDEZ, 2003). Os hábitos onívoros das formigas neste grupo e a ocorrência de quatro espécies desse gênero no ambiente de estudo são indicativos de processos iniciais de recuperação ecológica (LUTINSKI et al., 2017a). Este comportamento pode explicar a ocorrência da espécie Linepithema angulatum nos ambientes de APP, destacando-se a inexistência de registros prévios desta espécie para e estado do Rio Grande do Sul.

Espécies do gênero Nylanderia são consideradas generalistas e oportunistas, toleram ambientes abertos e desprovidos de vegetação (SILVESTRE; BRANDÃO; SILVA, 2003). A baixa frequência das espécies de Nylanderia nas amostras indica que as APP1 e 2 e as plantações de eucalipto já se encontram em um estágio avançado de sucessão ecológica. Formigas pertencentes 
ao gênero Pseudomyrmex ocorrem intimamente associadas à vegetação (BACCARO et al., 2015). A ocorrência da espécie Pseudomyrmex gracilis somente na APP1 corrobora os achados dos autores Baccaro et al. (2015) e, juntamente com as ocorrências restritas de Nylanderia, reforçam o estágio de conservação dos ambientes, especiamente das áreas de APP.

Espécies de Wasmannia são descritas como invasoras e tolerantes às perturbações ambientais. Juntamente com formigas do gênero Crematogaster possuem hábitos onívoros (SILVESTRE; BRANDÃO; SILVA, 2003). As formigas desses gêneros recrutam massivamente, possuem tamanho diminuto (SILVESTRE; BRANDÃO; SILVA, 2003) e dominam as fontes de alimentos o que pode explicar a ocorrência nas APPs (LUTINSKI et al., 2017c). Esses fatores podem explicar a ocorrencia variada das espécies desses gêneros nas quatro áreas.

Observou-se diferença significativa quanto à riqueza de formigas entre os ambientes amostrados (Figura 1). Observou-se maior riqueza para as assembleias de formigas dos ambientes APP1 e EUC1, enquanto a menor foi verificada para as assembleias dos ambientes APP2 e EUC2. Os menores valores de ocorrência foram registrados nos ambientes APP2 ( $\mathrm{n}=87)$ e EUC1 ( $\mathrm{n}=$ 86), enquanto os maiores foram observados na APP1 ( $n=111)$ e EUC2 ( $n=110)$.

Figura 1 - Comparação pelo método de rarefação, com base no número de ocorrências e na riqueza de assembleias de formigas nos quatro ambientes no município de Frederico Westphalen, Rio Grande do Sul, Brasil (2018).

Figure 1 - Comparison by the rarefaction method, based on the occurrence numbers and richness of ant assemblages in the four environments in the Frederico Westphalen municipality, Rio Grande do Sul state, Brazil (2018).

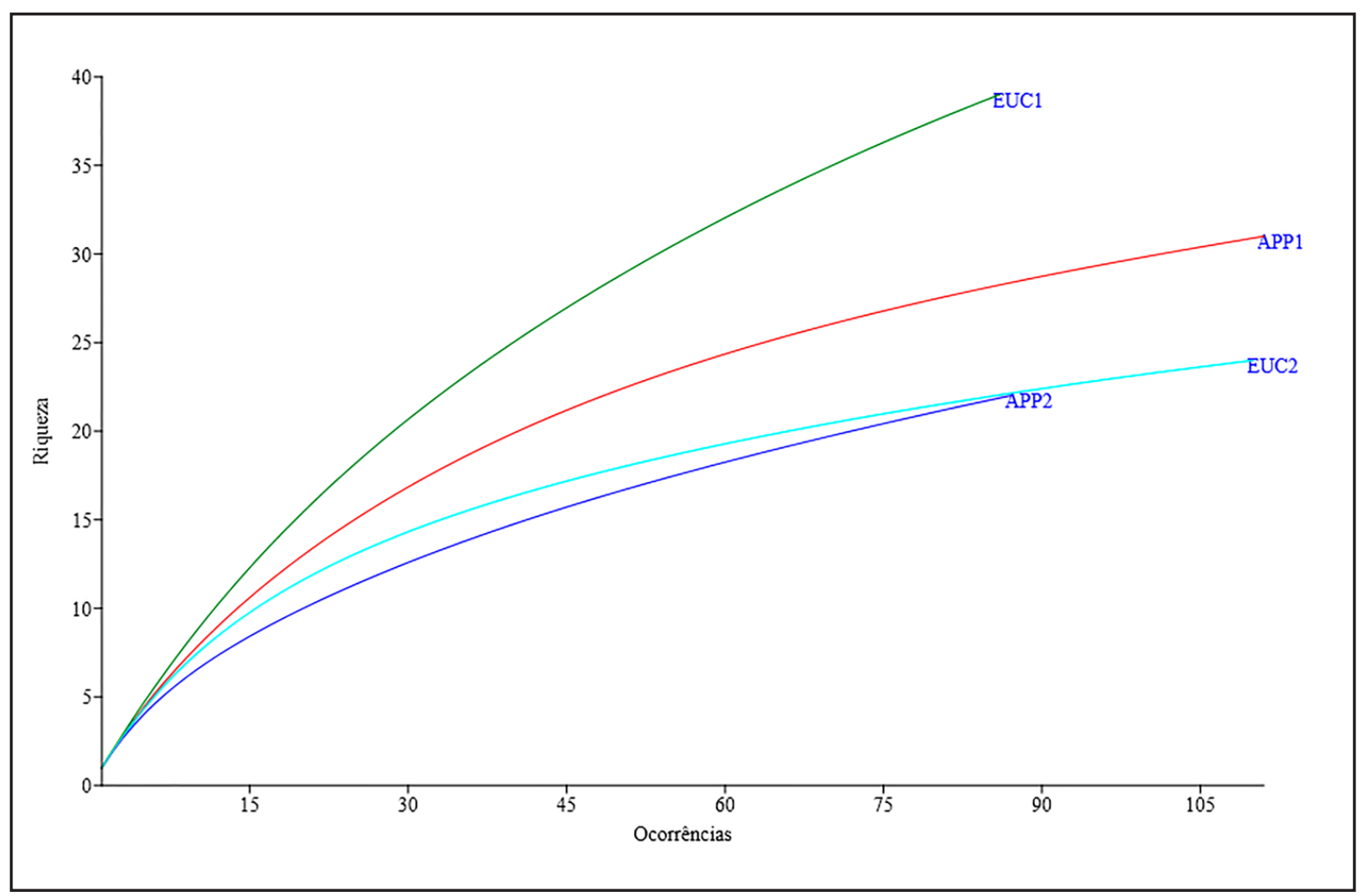

Fonte: Autores (2018)

Em que: APP1-2: Áreas de preservação permanente, 1 e 2; EUC1-2: Plantações de eucalipto, 1 e 2. 
Chama a atenção de que a maior riqueza tenha sido amostrada no ambiente EUC1, sendo este uma plantação de eucalipto. A diferença para a APP2 foi de 62,5\% e do ambiente EUC2 foi de 70,8\%. Tamanho das áreas, tempo de regeneração das APP, espaçamentos entre as árvores das plantações de eucalipto, a presença e a espessura da serrapilheira podem estar associadas à riqueza de formigas nos ambientes. Contudo, a realização de apenas um evento amostral pode ter sido determinante para a amostragem. Formigas sofrem a influência de fatores climáticos como a temperatura e a umidade (HÖLLDOBLER; WILSON, 1990). Segundo Lutinski et al. (2017b), a sazonalidade também interfere nos inventários de formigas. Esses fatores todos, combinados, podem ter influenciado e determinado a riqueza nos ambientes amostrados, apresentando um padrão diferente do esperado, já que nas APPs se esperava uma riqueza maior em relação às plantações de eucalipto.

A principal diferença entre os valores observados (Sobs) e a riqueza estimada (Chao 1) foi encontrada para as assembleias de formigas do ambiente APP2, 62,5\% (Chao $1=35,8$ ) e ambiente EUC2, 23,3\% (Chao 1= 29,6). No ambiente APP1, por sua vez, a diferença foi de 35,5\% (Chao $1=$ 42,0 ), enquanto no ambiente EUC1 foi de $48,7 \%$ (Chao 1= 58,0) (Tabela 2).

A assembleia de formigas do ambiente EUC1 apresentou maior riqueza $(S=39)$, seguida pela assembleia do ambiente APP1 $(S=31)$, ambiente EUC2 $(S=24)$ e APP2 $(S=22)$. A maior diversidade foi observada no ambiente EUC1 $\left(\mathrm{H}^{\prime}=3,40\right)$, enquanto a menor foi no ambiente APP2 $\left(H^{\prime}=2,48\right)$. O mesmo padrão foi observado para a uniformidade, com o maior valor registrado no ambiente EUC1 (J '=0,93) e o menor no ambiente EUC2 (J'=0,80) (Tabela 2).

Tabela 2 - Índices de diversidade para assembleias de formigas dos quatro ambientes amostrados, no município de Frederico Westphalen, Rio Grande do Sul, Brasil (2018).

Table 2 - Diversity indexes for ant assemblages from the four sampled environments, in Frederico Westphalen municipality, Rio Grande do Sul state, Brazil (2018).

\begin{tabular}{lccccc}
\hline \multicolumn{1}{c}{ Índices } & APP1 & APP2 & EUC1 & EUC2 & Média \\
\hline Sob(s) & 31 & 22 & 39 & 24 & 29 \\
Ocorrências & 111 & 87 & 86 & 110 & 98,5 \\
Shannon (H') & 3,02 & 2,48 & 3,40 & 2,77 & 2,92 \\
Equitabilidade (J') & 0,88 & 0,80 & 0,93 & 0,87 & 0,87 \\
Chao 1 & 42,0 & 35,8 & 58,0 & 29,6 & 41,3 \\
Diferença Sob(s) X Chao 1 (\%) & 35,5 & 62,5 & 48,7 & 23,3 & 42,5 \\
\hline
\end{tabular}

Em que: APP1-2: Áreas de preservação permanente, 1 e 2; EUC1-2: Plantações de eucalipto, 1 e 2.

O índice de diversidade de Shannon foi de 3,$02 ; 2,48 ; 3,40$ e 2,77 e a equitabilidade foi de 0,$88 ; 0,80 ; 0,93$ e 0,87 para os ambientes APP1, APP2, EUC1 e EUC2, respectivamente. A diversidade de Shannon para o ambiente APP2 pode estar associada à circulação de pessoas no ambiente, impactando assim a riqueza e a abundância de formigas. Lavouras de milho e de soja no entorno do ambiente APP2 também podem estar interferindo na assembleia de formigas, uma vez que se trata de lavouras convencionais em que é frequente o uso de agrotóxicos como herbicidas e inseticidas. A assembleia do ambiente EUC2 pode ter sido influenciada pelo tamanho do fragmento comparado aos demais ambientes amostrados. O maior valor encontrado para a equitabilidade foi para o ambiente EUC1, indicando uma distribuição mais uniforme da fauna de formigas neste ambiente. Segundo Pinto-Coelho (2000), o índice de equitabilidade é 
considerado indicativo de uniformidade na distribuição das espécies no local avaliado e esse equilíbrio contribui positivamente para o estabelecimento de comunidades mais diversificadas.

A abundância e a composição apresentaram grupos similares nas amostras. Um agrupamento formado por APP1s, EUC1s, EUC2s e EUC2g e o grupo APP2g, APP2s, APP1p, EUC2p e APP2p apresentaram similaridade de $40 \%$ entre si. Já os APP1g, EUC1g e EUC1p diferiram entre si e em relação aos demais ambientes e métodos amostrados (Figura 2).

O padrão de similaridade entre amostras obtidas pelos diferentes métodos de amostragem, combinados com os ambientes, demonstrou ser aleatório, já que áreas de APP, plantações de eucaliptos e os métodos não se aproximaram de acordo com o esperado. Isso pode estar associado à presença de sub-bosque e a presença de serapilheira no ambiente EUC1 e nas APP1 e 2, corroborando os resultados encontrados por Cantarelli et al. (2015). Ambientes mais complexos estruturalmente podem manter maior diversidade e riqueza por apresentarem maiores possibilidades de abrigo, reprodução, nidificação e alimentação, além de maior estabilidade (ILHA et al., 2009). Contudo, assim como na riqueza, o evento amostral único associado com as condições de temperatura e de unidade específicas de cada dia em que os ambientes foram visitados para as amostras, podem explicar melhor a similaridades apresentadas.

Figura 2 - Similaridade entre as assembleias de formigas amostradas em quatro ambientes e os métodos utilizados, no município de Frederico Westphalen, Rio Grande do Sul, Brasil (2018).

Figure 2 - Similarity among the assemblages of ants sampled in four environments and the methods used, in Frederico Westphalen, Rio Grande do Sul state, Brazil (2018).

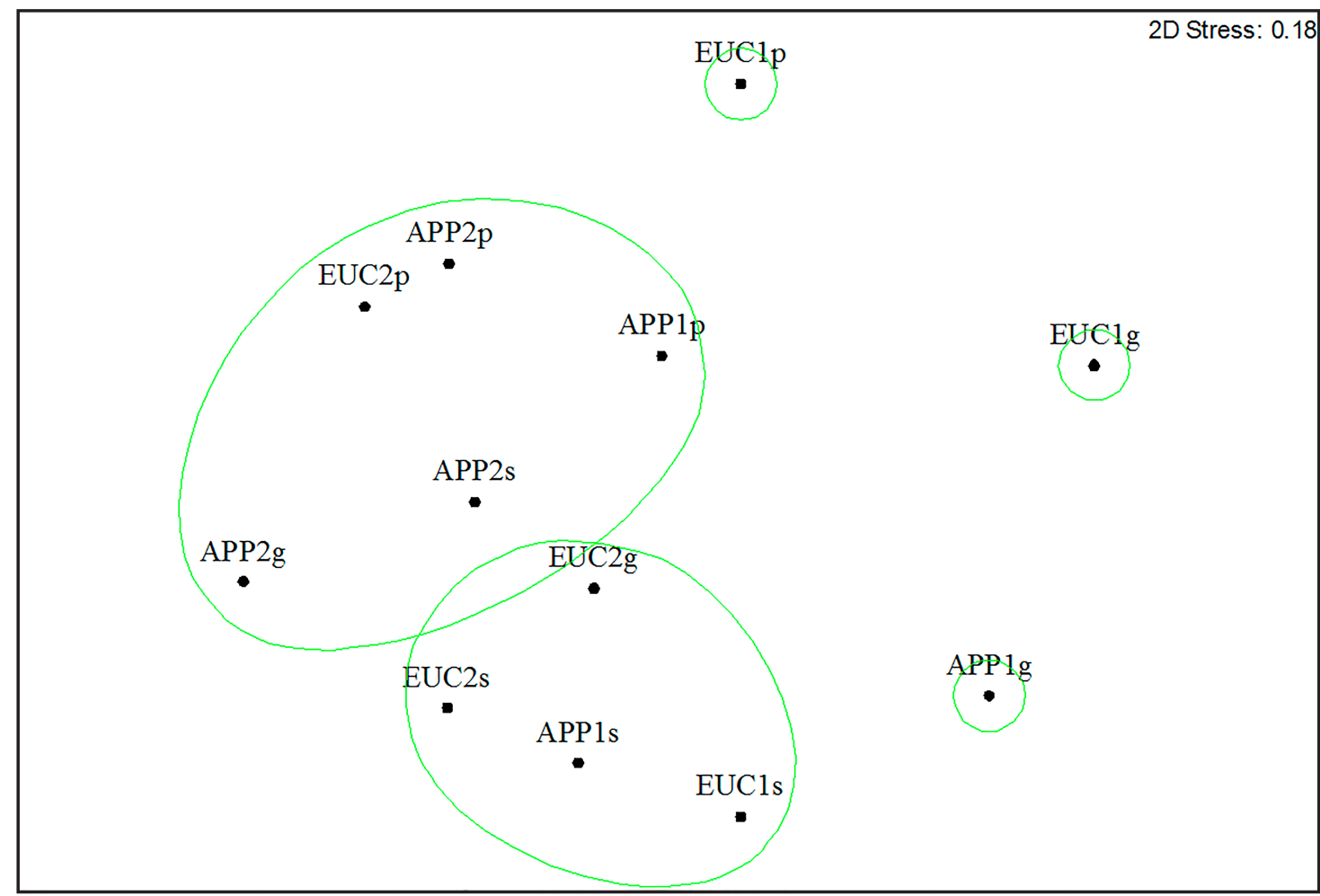

Fonte: Os autores (2018)

Em que: APP1g: ambiente 1, glicose; APP1s: ambiente 1, sardinha; APP1p: ambiente 1, pitfall; APP2g: ambiente 2, glicose; APP2s: ambiente 2, sardinha; APP2p: ambiente 2, pitfall; EUC1g: ambiente 3, glicose; EUC1s: ambiente 3, sardinha; EUC1p: ambiente 3, pitfall; EUC2g: ambiente 4, glicose; EUC2s: ambiente 4, sardinha; EUC2p: ambiente 4, pitfall. 
Ao todo, 76,4\% da variação na ocorrência de formigas, de acordo com os ambientes amostrados, foram explicados pelos dois primeiros componentes da PCA. Quatro espécies apresentaram associação com as amostragens feitas nos ambientes APP2 e EUC1 (Pachycondyla striata, Pheidole dyctiota, Pheidole pubiventris e Pheidole sp. 8). Oito espécies ocorreram associadas às amostragens dos ambientes APP1 e EUC2 (Acromyrmex subterraneus, Brachymyrmex aphidicola, Linepithema angulatum, Pheidole aberrans, Pheidole megacephala, Pheidole risii, Pheidole sp. 1, Pheidole sp. 12). Todas as demais espécies de formigas ocorreram independentemente do tipo de ambiente (Figura 3).

Figura 3 - Análise de Componentes Principais (PCA) das espécies de formigas dos quatro ambientes amostradas no município de Frederico Westphalen, Rio Grande do Sul, Brasil (2018).

Figure 3 - Principal Component Analysis (PCA) of the ant species of the four environments sampled in Frederico Westphalen, Rio Grande do Sul, Brazil (2018).

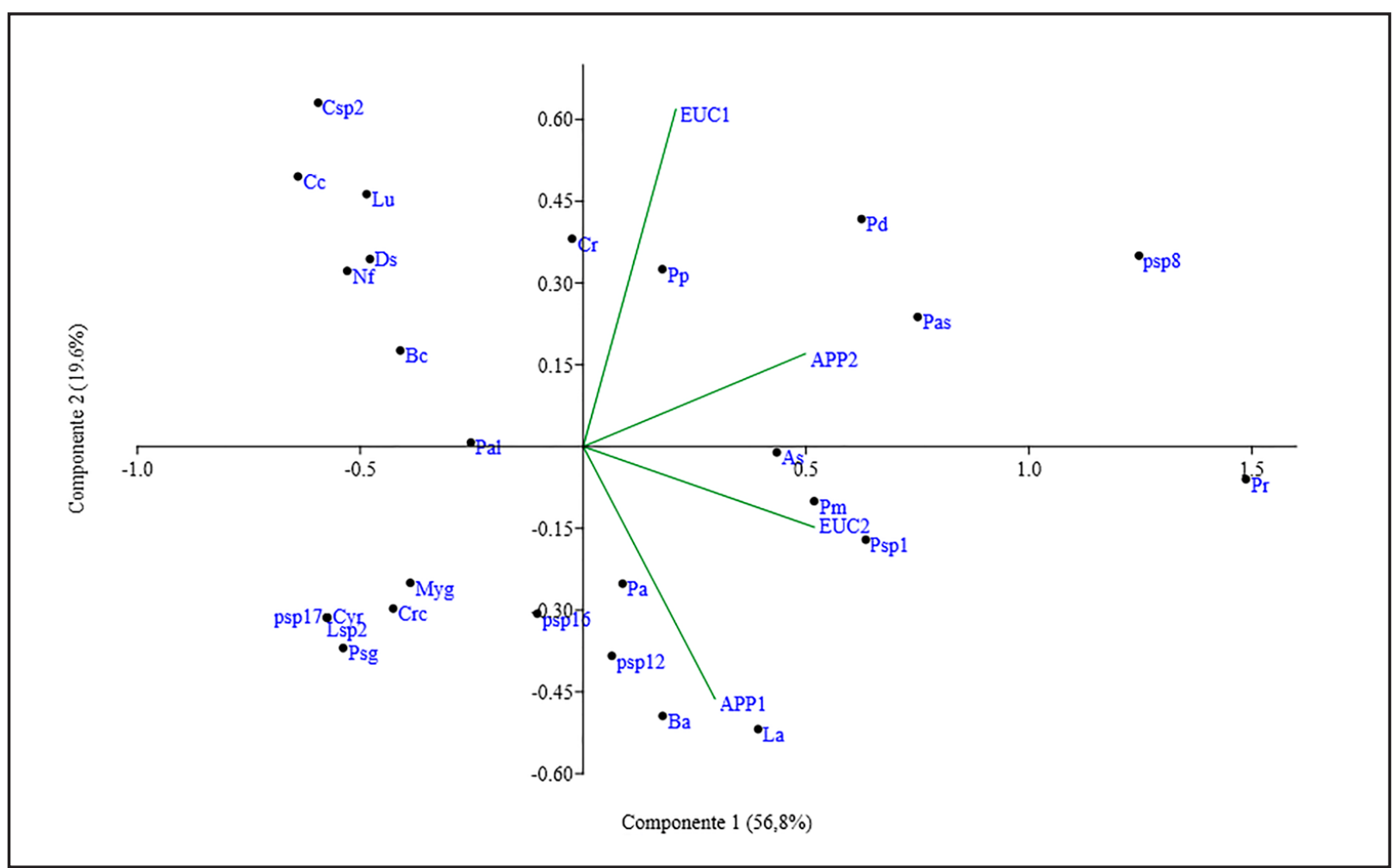

Fonte: Autores (2018)

Em que: APP1-2: Áreas de preservação permanente, 1 e 2; EUC1-2: Plantações de eucalipto, 1 e 2. As: Acromyrmex subterraneus; Ba: Brachymyrmex aphidicola; Bc: Brachymyrmex coactus; Cc: Camponotus crassus; Cr: Camponotus rufipes; Crc: Crematogaster corticicola; Csp2: Camponotus sp. 2; Cyr: Cyphomyrmex rimosus; Ds: Diplorhoptrum strictum; La: Linepithema angulatum; Lsp2: Linepithema sp. 2; Lu: Linepithema humile; Myg: Mycocepurus goeldii; Nf: Nylanderia fulva; Pa: Pheidole aberrans; Pal: Paratrechina longicornis; Pas: Pachycondyla striata; Pd: Pheidoledyctiota; Pm: Pheidole megacephala; Pp: Pheidolepubiventris; Pr: Pheidole risii; Psg: Pseudomyrmex gracilis; Psp1: Pheidole sp. 1; Psp8: Pheidole sp. 8; Psp12: Pheidole sp.12; psp16: Pheidole sp. 16; psp17: Pheidole sp. 17.

A ocorrência das formigas em um determinado ambiente pode ser definida por vários fatores bióticos e abióticos (HÖLLDOBLER; WILSON, 1990). A harmonia destes fatores é um condicionante para a ocorrência das formigas nos ambientes amostrados, já que foi observada uma similaridade na composição e abundância das assembleias de formigas de $40 \%$ em nove dos 12 conjuntos de amostras. 


\section{Conclusão}

O estudo apresenta uma lista com 71 espécies de formigas para uma região do estado do Rio Grande do Sul ainda pouco amostrada quanto à mirmecofauna. Apesar de a riqueza, abundância, diversidade e equitabilidade apontarem diferenças entre os ambientes amostrados, parte da diferença pode estar associada à amostragem e às variações climáticas intrínsecas dos eventos amostrais, em cada ambiente. Amostras adicionais em ambientes similares na região poderão contribuir para ampliar o conhecimento da mirmecofauna e para a compreensão dos fatores associados.

Contudo, cabe destacar que os resultados desta pesquisa poderão ser usados para o conhecimento e a atualização das ocorrências de formigas no estado do Rio Grande do Sul, além de servirem como base para a construção de planos de manejo e da conservação da fauna de invertebrados, considerando áreas de APP e plantios de eucalipto.

\section{Agradecimentos}

Aos proprietários da área de estudo pela autorização para realização das amostras. À Unochapecó pela Bolsa de Produção Cientifica. Ao ICMBio pela licença para a realização da pesquisa.

\section{Referências}

ALVARES, C. A. et al. Köppen's climate classification map for Brazil. Meteorologische Zeitschrift, Stuttgart, v. 22, n. 6, p. 711-728, jan. 2014.

BACCARO, F. B. et al. Guia para os gêneros de formigas do Brasil. Manaus: INPA, 2015. 388 p.

BESTELMEYER, B. T. et al. Field techniques for the study of ground-dwelling ants. In: AGOSTI, D. et al. (ed.). Ants: standard methods for measuring and monitoring biodiversity. Washington: Smithsonian Institution Press, 2000. p. 122-144.

BHARTI, H.; BHARTI, M.; PFEIFFER, M. Ants as bioindicators of ecosystem health in Shivalik Mountains of Himalayas: assessment of species diversity and invasive species. Asian Myrmecology, Ulaanbaatar, v. 8, p. 1-15, dez. 2016.

CANTARELLI, E. B. et al. Diversidade de formigas (Hymenoptera: Formicidae) da serrapilheira em diferentes sistemas de uso do solo. Ciência Florestal, Santa Maria, v. 25, n. 3, p. 607-616, set. 2015.

CLARKE, K. R.; GORLEY, R. N. Primer: Getting started with v6. Plymouth routines in multivariate ecological research. [S. l.]: PRIMER-E, 2005.

COSTA-MILANEZ, C. B. et al. Are ant assemblages of Brazilian veredas characterised by location or habitat type? Brazilian Journal of Biology, São Carlos, v. 74, n. 1, p. 89-99, 2014.

CREPALDI, R. A. et al. Formigas como bioindicadores da qualidade do solo em sistema integrado lavoura-pecuária. Ciência Rural, Santa Maria, v. 44, n. 5, p. 781-787, maio 2014.

EDWARDS, D. P. et al. Land-sharing versus land-sparing logging: reconciling timber extraction with biodiversity conservation. Global Change Biology, Londres, v. 20, p. 183-191, nov. 2014.

FERNANDEZ, F. Introducción a las hormigas de la región neotropical. Bogotá: Instituto de Investigación de Recursos Biológicos Alexander Von Humboldt, 2003. 418 p.

GOTELLI, N. J.; ENTSMINGER, G. L. EcoSim: null models software for ecology. Versão 7.0. [S. l.]: Acquired Intelligence Inc. \& Kesey-Bear, 2001.

HAMMER, O.; HARPER, D. A. T.; RYAN, P. D. Past: palaeonthological statistics software package for education and data analysis. Version. 1.37. [S. l.: s. n.], 2001. 
HOLDEFER, D. R.; LUTINSKI, J. A.; GARCIA, F. R. M. Does organic management of agroecosystems contribute to the maintenance of the richness of ants? Semina: Ciências Agrárias, Londrina, v. 38, n. 6, p. 3455-3468, nov./dez. 2017.

HÖLLDOBLER, B.; WILSON, E. O. The ants. Cambridge: Harvard University Press, 1990. 732 p.

IBGE. Cidades. Rio de Janeiro, 2016. Disponível em: http://www.ibge.gov.br. Acesso em: 01 maio 2017.

ILHA, C. et al. Riqueza de formigas (Hymenoptera: Formicidae) da Bacia da Sanga Caramuru, município de Chapecó-SC. Biotemas, Florianópolis, v. 22, n. 4, p. 95-105, dez. 2009.

LUTINSKI, J. A. et al. Ant assemblages (Hymenoptera: Formicidae) associated to environments of a rural property in the extreme Western Region of the State of Santa Catarina. Revista Brasileira de Ciências Ambientais - RBCIAMB, Rio de Janeiro, n. 47, p. 12-23, mar. 2018.

LUTINSKI, J. A. et al. Ant assemblage (Hymenoptera: Formicidae) in three wind farms in the State of Paraná, Brazil. Brazilian Journal of Biology, São Carlos, v. 77, n. 1, p. 176-184, jan./mar. 2017a.

LUTINSKI, J. A. et al. Efeitos microclimáticos e temporais sobre a assembleia de formigas (Hymenoptera: Formicidae) de áreas urbanas do Oeste de Santa Catarina: efeitos microclimáticos sobre comunidades de formigas. In: LUTINSKI, J. A. (org.). Formigas em ambientes urbanos de Santa Catarina. Curitiba: CRV, 2017b.

LUTINSKI, J. A. et al. Richness and structure of ant assemblies (Hymenoptera: Formicidae) in Atlantic forest in southern Brazil. Annals of the Brazilian Academy of Sciences, Rio de Janeiro, v. 89 , n. 4 , p. 2719-2729, out./dez. 2017c.

LUTINSKI, J. A.; LOPES, B.; MORAIS, A. B. B. Diversidade de formigas urbanas (Hymenoptera: Formicidae) de dez cidades do sul do Brasil. Biota Neotropica, Campinas, v. 13, n. 3, p. 332342, set. 2013.

MADEIRA, B. G. et al. Changes in tree and Liana communities along a successional gradient in a tropical dry forest in south-eastern Brazil. Plant Ecology, Netherland, v. 201, p. 291-304, jan. 2009.

NAYANA, P. et al. Comparison of ant (Hymenoptera: Formicidae) diversity in different habitats of Machad Region of Thrissur. Bulletin of Environment, Pharmacology and Life Sciences, Agra, v. 5, n. 2, p. 28-33, jan. 2016.

PINTO-COELHO, R. M. Fundamentos em ecologia. Porto Alegre: Artmed, 2000. 252 p.

ROCHA, W. O. et al. Formigas (Hymenoptera: Formicidae) bioindicadoras de degradação ambiental em Poxoréu, Mato Grosso, Brasil. Floresta e Ambiente, Seropédica, v. 22, n. 1, p. 88-98, mar. 2015.

ROMERO, H.; JAFFE, K. A comparison of methods for sampling ants (Hymenoptera: Formicidae) in Savanna. Biotropica, Hoboken, v. 21, p. 348-352, 1989.

SILVESTRE, R.; BRANDÃO, C. R. F.; SILVA, R. R. da. Grupos funcionales de hormigas: el caso de los gremios del Cerrado. In: FERNANDEZ, F. (ed.). Introducción a las hormigas de la región neotropical. Bogotá: Instituto de Investigación de Recursos Biológicos Alexander von Humboldt, 2003, p. 113-148, 2003.

TUNDISI, J. G.; TUNDISI, T. M. Impactos potenciais das alterações do Código Florestal nos recursos hídricos. Biota Neotropica, Campinas, v. 10, n. 4, p. 67-76, dez. 2010.

ULYSSÉA, M. A. et al. Updated list of ant species (Hymenoptera, Formicidae) recorded in Santa Catarina State, southern Brazil, with a discussion of research advances and priorities. Revista Brasileira de Entomologia, Curitiba, v. 55, n. 4, p. 603-611, dez. 2011.

WIELGOSS, A. et al. Interaction complexity matters: disentangling services and disservices of ant communities driving yield in tropical agroecosystems. Proceeding of Royal Society B, United Kingdom, v. 281, n. 1775, p. 1-10, jan. 2014. 